\title{
The Relationship between the Occipitocervical Junction and Thoracic Kyphosis in Ankylosing Spondylitis: A Retrospective Cohort Study of 86 Cervical Fractures in Surgically Treated Patients
}

\author{
Nodoka Manabe ${ }^{1,2}$, Augusto Covaro ${ }^{1,3}$, Lukas Bobinski ${ }^{1}$, \\ Takachika Shimizu ${ }^{2}$, Claes Olerud ${ }^{1}$, Yohan Robinson ${ }^{1}$ \\ ${ }^{I}$ Department of Surgical Science, Uppsala University Hospital, Uppsala, Sweden \\ ${ }^{2}$ Department of Orthopedic Surgery, Gunma Spine Center, Harunaso Hospital, Takasaki, Japan \\ ${ }^{3}$ Department of Orthopedic Surgery, Hospital Universitari Dexeus, Barcelona, Spain
}

Study Design: Retrospective analysis of prospectively collected data.

Purpose: To describe the radiological characteristics of the occipitocervical area in patients with ankylosing spondylitis (AS) using the novel measure $X$-angle and to describe the correlation between the ankylosed occipitoatlantoaxial (OAA) joint and thoracic kyphosis (TK).

Overview of Literature: AS affects the axial skeleton, leading to progressive ankylosis of all vertebral segments. The effect of ankylosis on the upper cervical area of these patients is not well documented.

Methods: All patients with complete ankylosis of the spinal column between C3 and T1, treated for cervical spinal fracture between 2007 and 2014, were eligible for inclusion in this study. The level of cervical fracture was identified. The 11-12 and T5-12 angles were measured using preoperative lateral radiography. The progressive degeneration of the C0-C1-C2 joints was evaluated via the new indicator $\mathrm{X}$-angle, through the measurement of the angle of the C0-C1-C2 articulations in the coronal plane using computed tomography.

Results: We included 86 consecutive patients with AS (67 males) aged 69 12 years. The patients were divided into two groups according to the degenerative change in the $\mathrm{CO}-\mathrm{C} 1$ joint (62 patients with a mobile joint and 24 patients with an ankylosed joint). There was no significant difference between the two groups in terms of age $(p=0.094)$ and level of fracture $(p=0.949)$. The most commonly affected level was $\mathrm{C} 6$. There was no requirement for revision due to non-union in any of the patients. There was a statistically significant difference observed in the T1-12, T5-12, and $X$-angles $(p=0.004,0.001$, and $<0.001$, respectively). TK was greater in the ankylosed joint group than in the mobile joint group. The $\mathrm{X}$-angle was also greater in the ankylosed joint group because of the vertical destruction of the OAA joint.

Conclusions: Thoracic hyperkyphosis resulted in degenerative changes in the C0-C1-C2 joint in patients with AS. The X-angle is a reliable method for measuring the integrity of the C0-C1-C2 joint in such patients.

Keywords: Ankylosing spondylitis; Spinal fracture; Atlanto-occipital joint; Kyphosis

Received Jan 9, 2018; Revised May 15, 2018; Accepted Jun 3, 2018

Corresponding author: Nodoka Manabe

Department of Orthopaedic Surgery, Gunma Spine Center, Harunaso Hospital, 828-1, Kamitoyooka, Takasaki, Gunma, 370-0871, Japan Tel: +81-27-343-8000, Fax: +81-27-343-6622, E-mail: nodokamanabe0817@me.com 


\section{Introduction}

Ankylosing spondylitis (AS) is a common inflammatory rheumatic disease affecting the axial skeleton and leading to progressive ankylosis of all spinal segments from the occiput to the iliac bone. Because of reduced biomechanical flexibility, the spinal column acts like a long-bone lever, causing severe instability in cases with vertebral fractures. Cervical and cervicothoracic fractures related to AS are common and potentially hazardous. These patients may experience implant failure, pseudarthrosis, and dislocation. In addition, the mortality rate among patients with such fractures is considerably high [1-5]. Recently, the use of antirheumatic treatment for AS has improved the course of the disease considerably. However, the frequency of cervical fractures related to AS is increasing progressively $[6,7]$.

AS leads to total immobility of the spinal column and often fused thoracic hyperkyphosis [8], compromising the ability of the patients to sit, stand, or lie smoothly. Thoracic hyperkyphosis causes hyper-cervical lordosis because patients acquire a horizontal gaze $[9,10]$. Furthermore, patients with AS are unable to see the horizon, causing severe problems in daily activities. The fusion of the occipitocervical junctions is usually delayed in patients with AS. Although commonly observed, the progression of ankylosis in the upper cervical spine is not well documented.

In this study we present the radiographic features of the occipitoatlantoaxial (OAA) joint in patients with AS using a novel measure termed $\mathrm{X}$-angle [11] and describe the correlation between the ankylosed OAA joint and thoracic kyphosis (TK).

\section{Materials and Methods}

\section{Study design}

This was a retrospective cohort study of prospectively collected data from patients with AS assessed for spinal fracture. The study was performed in the Uppsala University Hospital and approved by the Uppsala regional ethical review board (IRB approval no., 2010/131, no. 2010/297/1) and complies with the criteria of the STROBE (Strengthening the Reporting of Observational Studies in Epidemiology) and RECORD (Reporting of Studies Conducted Using Observational Routinely-Collected Health Data) statements for observational studies [12].

\section{Setting}

The study was performed in a single level-1 trauma-center at the Uppsala University Hospital, Uppsala, Sweden between 2007 and 2014. All patients with a history of AS and minor trauma/inflammatory back pain underwent investigation of the axial skeleton using computed tomography (CT) to exclude AS-related fractures or discovertebral (Andersson) lesions. Patients with spinal fractures treated surgically were registered in the Swedish Spine Registry (SWESPINE) [13]. Surgical treatment included long posterior fixation using a screw-rod construct extending three levels cranially to three levels caudally of the injury. In case of spinal cord injury, additional decompression and evacuation of epidural hematoma were performed (Fig. 1).

\section{Patients}

Patients meeting all the following criteria were included in the study: (1) AS with thoracic ankylosis; (2) acute cervical fracture; and (3) age $\geq 45$ years. Patients meeting any of the following criteria were excluded from the study: (1) incomplete radiographic examination (i.e., no inclusion of the craniocervical junction); (2) incomplete electronic record file of the patient; (3) age <45 years; (4) acute thoracic fractures; (5) acute lumbar fractures; and (6) living in a nursing home for patients with dementia.

Spinal fracture related to ankylosing spondylitis 2007-2014 (n=155)

69 Excluded due to following reasons: (1) thoracic and lumbar spinal fracture and (2) missing data

$\checkmark$ 86 Study population Subgroup analysis according to $\mathrm{CO}-\mathrm{C} 1$ joint integrity

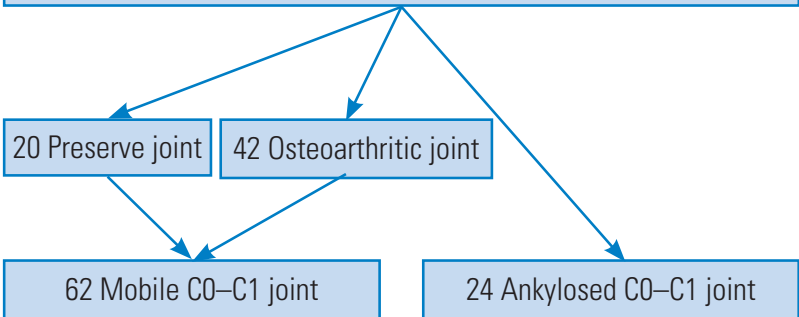

Fig. 1. Flow chart of patient inclusion. 


\section{Variables}

1) Classification of ankylosing spondylitis fractures Fractures were classified into four groups using the classification of AS introduced by Caron et al. [14], based on fracture excursion through the intervertebral disc injury (i.e., type I, transdisc disruption; type II, vertebral body injury; and types III and IV, combination of disc and vertebral body injuries) (Fig. 1).

\section{2) Thoracic sagittal alignment}

TK was measured preoperatively using the global Cobb angles T1-T12 and T5-T12 on lateral radiography. A line was drawn along the plane of the superior endplate of T1 or T5 and the inferior endplate of T12.

3) Osteoarthritis occipitoatlantoaxial joint changes: descriptive radiology and $\mathrm{X}$-angle

A sagittal view of the OAA joint was obtained prior to surgery using CT. The narrowing and/or sclerosis on the C0-C1 joint space, observed through CT, were defined as osteoarthritic changes. The disappearance and/or fusion of the $\mathrm{C} 0-\mathrm{C} 1$ joint space were defined as ankylosed joint.

The progressive degeneration of the $\mathrm{C} 0-\mathrm{C} 1-\mathrm{C} 2$ joints was evaluated using a new indicator termed $\mathrm{X}$-angle. The $\mathrm{X}$-angle is formed by lines drawn from the occipitoatlantal to the atlantoaxial joints on the contralateral side of coronal CT reconstructions, using the coronal plane perpendicular to the center of the $\mathrm{C} 1$ inferior articular surface (Fig. 2).

\section{Data sources and measurement}

All CT scans were analyzed using the multiplanar reconstruction function of the electronic radiographic informa-
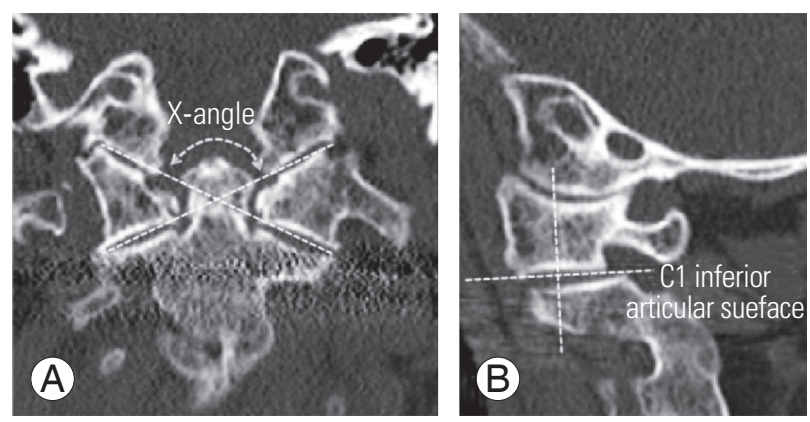

Fig. 2. (A, B) Landmarks for the $X$-angle measurement using the multiplanar reconstruction of a cervical computed tomography scan. tion system (Carestream PACS, Solna, Sweden). Change in the osteoarthritic OAA joint and the level of cervical fracture were assessed by three experienced investigators (NM, AC, and YR).

\section{Bias}

The inter- and intra-observer reliability of the $\mathrm{X}$-angle was assessed. The X-angle was measured independently by two investigators, and the intraclass correlation coefficient (ICC) and interclass correlation coefficient were determined.

\section{Statistical methods}

Subgroup analysis was performed according to the integrity of the $\mathrm{C} 0-\mathrm{C} 1$ joint. The mobile joint group was characterized by a preserved joint with osteoarthritic changes, while the ankylosed joint group exhibited fused articulations.

The non-paired sample $t$-test and Fisher's exact test were used to identify differences between groups. The ICC two-way mixed model on absolute agreement was used to analyze the reliability of X-angle measurements [15]. This is a ratio of the variance between patients to the total variance (patients, raters, and error). The values of the ICC range from 0 to 1 , with a higher value indicating better reliability. The ICC were interpreted as poor $(<0.40)$; fair ( 0.40 to 0.59$)$; good ( 0.60 to 0.74$)$, and excellent ( 0.75 to 1.00) [16].

All analyses were performed using The IBM SPSS ver. 22.0.0 for Mac (IBM Corp., Armonk, NY, USA). The mean values are presented with standard deviation, followed by intervals in brackets. All reported $p$-values were two-tailed, with $p<0.05$ denoting statistically significant differences.

\section{Results}

\section{Patients}

Patients with AS $(n=155)$ underwent spinal fixation for the treatment of spinal fracture between 2007 and 2014 . Of those, 69 patients were excluded from the analysis. The inclusion flow chart is presented in Fig. 1. We included 67 male and 19 female patients, aged $69.2 \pm 11.8$ years (range, 49-94 years), with AS and ankylosis of the thoracic spine. 

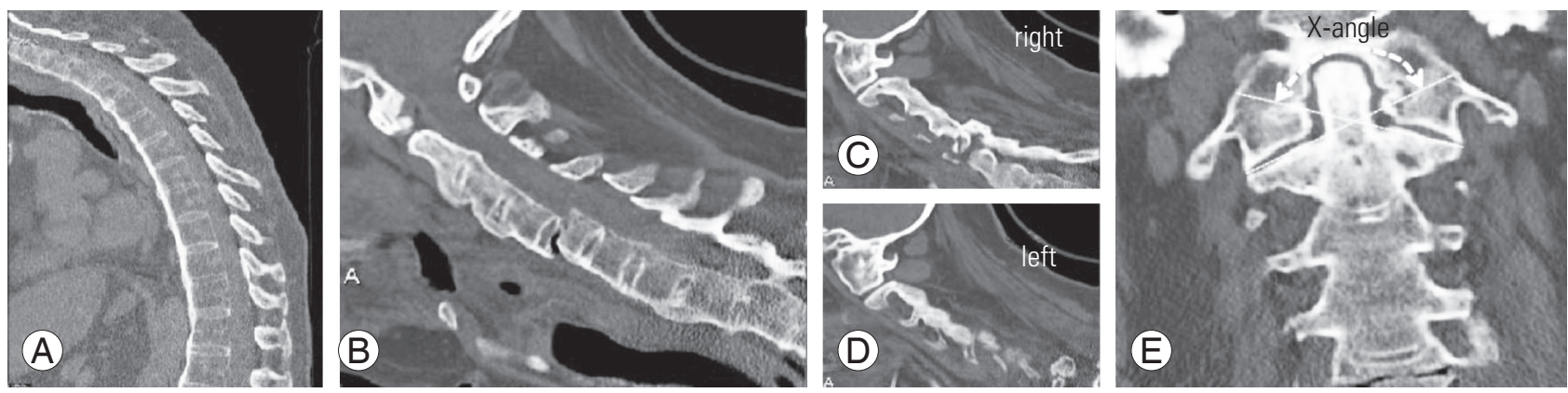

Fig. 3. A 56-year-old male patient suffered a low-energy external injury. He presented with a completely fused hyperkyphotic thoracic spinal column: (A) the T1-12 angle was $76^{\circ}$, and the T5-12 angle was $42^{\circ}$. (B-D) Injury computed tomography revealed a type 2 fracture (Caron et al. [14] classification) and a C5 fracture resembling a long-bone fracture. (C-E) The C0-C1 joint was completely ankylosed. However, the C1-C2 joint was not ankylosed. The $\mathrm{X}$-angle was $139^{\circ}$ with vertical distraction of the occipitoatlantal joints.
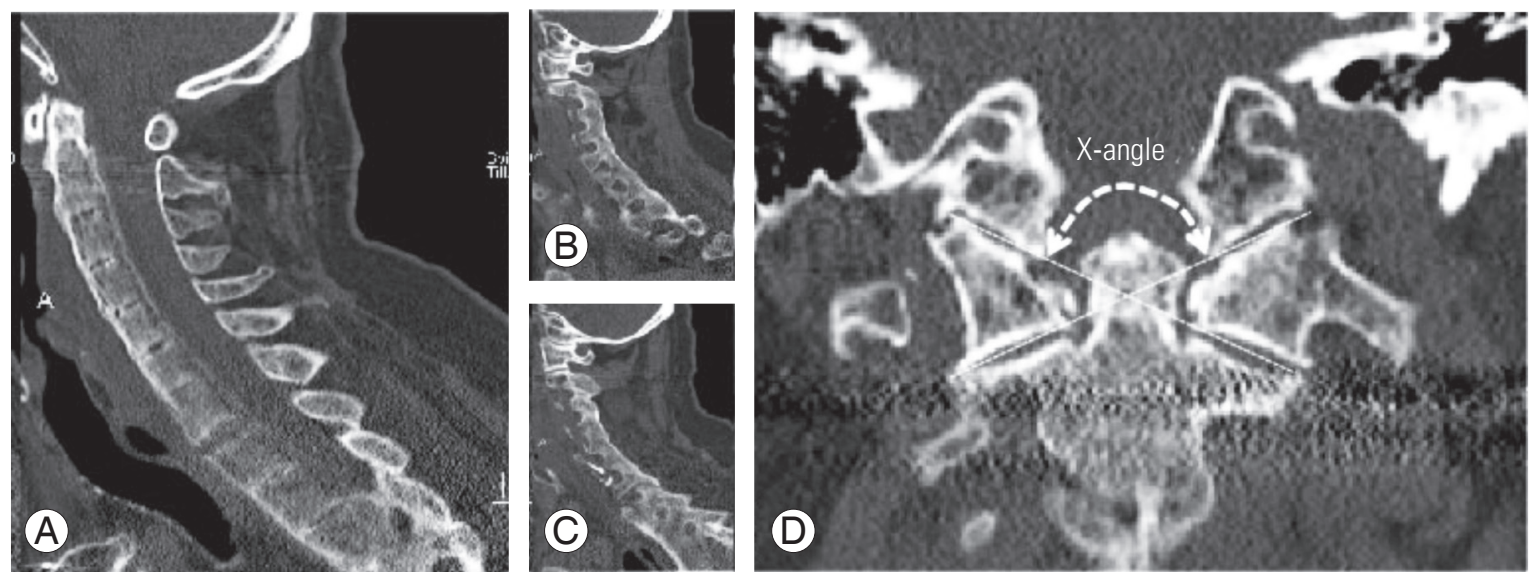

Fig. 4. A 73-year-old male patient presented with a C7-Th1 fracture: (A) type 1 fracture (Caron et al. [14] classification). (B-D) Injury computed tomography revealed a non-fused C0-C1-C2 joint. The X-angle was $125^{\circ}$ with symmetrical osteoarthritis of the occipitoatlantal joints.
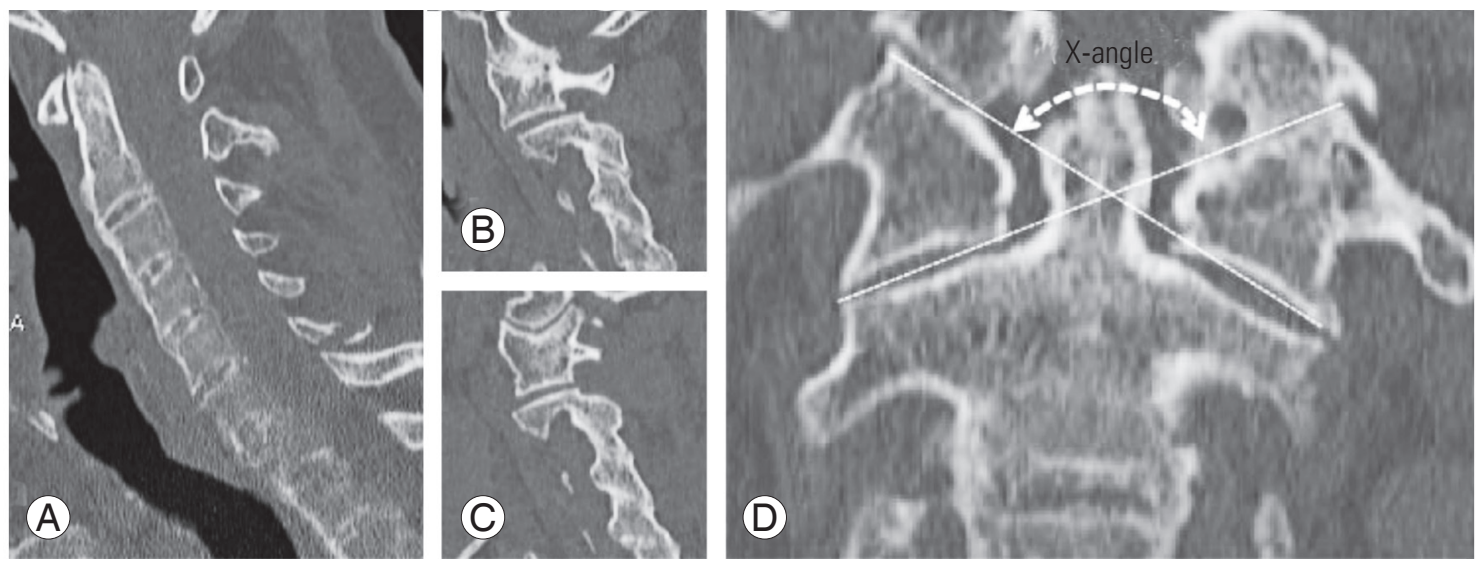

Fig. 5. (A) A 70-year-old male patient presented with a type 2 (Caron et al. [14] classification) C7 fracture. (B-D) Injury computed tomography revealed $\mathrm{CO}-\mathrm{C1}$ osteoarthritis and non-fused $\mathrm{CO}-\mathrm{C} 1$ on the right and left sides of the joint, respectively. In this case, defining the morphological change in the $\mathrm{CO}-\mathrm{C} 1$ joint was challenging, because the right side of the $\mathrm{CO}-\mathrm{C} 1$ joint had osteoarthritis or fused joint, whereas the left side of the joint was not fused. (D) However, the X-angle was measurable $\left(129^{\circ}\right)$, with asymmetrical vertical distraction of the occipitoatlantal joints and was slightly greater than that observed in the mobile group. 
Representative cases are shown in Figs. 3-5.

\section{Descriptive data}

A total of 78 patients underwent posterior fixation surgery, while eight patients underwent combined anterior and posterior fixation surgery. Posterior screws were inserted unless they were localized in three vertebrae cranially and three vertebrae caudally from the injury. Of the 86 patients with AS, three patients in the fused joint group were treated with posterior stabilization from the occipital bone to the upper thoracic spine using a screw-rod system (Table 1).

\section{Main results}

The 86 patients with AS were divided into subgroups according to the integrity of their $\mathrm{C} 0-\mathrm{C} 1$ joint: the mobile

Table 1. Demographic and clinical characteristics of 86 ankylosing spondylitis patients who underwent cervical posterior fusion surgery for cervical fracture

\begin{tabular}{lc}
\hline Characteristic & Value \\
\hline Total no. of patients & 86 \\
\hline Sex (male) & $67(78)$ \\
\hline Age at surgery (yr) & $69.2 \pm 11.8$ \\
\hline Weight (kg) & $75 \pm 12$ \\
\hline Height (cm) & $169 \pm 8$ \\
\hline Level of fracture & \\
\hline C2 & $4(5)$ \\
\hline C3 & $2(2)$ \\
\hline C4 & $7(8)$ \\
\hline C5 & $14(16)$ \\
\hline C6 & $33(38)$ \\
\hline C7 & $26(30)$ \\
\hline Caron classification & \\
\hline Type 1 & $38(44)$ \\
\hline Type 2 & $36(42)$ \\
\hline Type 3 & $9(10)$ \\
\hline Type 4 & $3(3)$ \\
\hline Purgical technique & $78(91)$ \\
\hline Antero-posterior fixation & $8(9)$ \\
\hline
\end{tabular}

Values are presented as number of patients (\%) or mean \pm standard deviation, unless otherwise stated. joint group included 20 patients with a preserved joint and 42 patients with osteoarthritic changes, while the ankylosed joint group included 24 patients with fused articulations (Fig. 1). There was no significant difference observed between the two groups in terms of age and the level of fracture. A C6 vertebral fracture was the most common fracture in the present study. There was a statistically significant difference observed in the T1-T12 and T5-T12 angles. TK was greater in the fused group than in the mobile joint group. The most significant difference between the two groups was recorded using the X-angle. The X-angle was greater in the ankylosed joint group because of the vertical destruction of the OAA joint (Table 2).

\section{Other analyses: intra- and inter-observer reliability of the $\mathrm{X}$-angle measurements}

The ICC and interclass correlation coefficient $r$ values for the relationship between the two investigators were 0.945 $(p<0.001)$ and $0.926(p<0.001)$, respectively (Table 3$)$.

\section{Discussion}

\section{Key results}

This was the first radiographical cohort study employing the X-angle method in patients with an ankylosed spine related to AS. The X-angle had high inter-observer reliability and could be related to thoracic sagittal alignment.

\section{Limitations}

Patients with AS underwent radiological imaging testing

Table 2. Demographic and clinical characteristics of two groups according to $\mathrm{CO}-\mathrm{C} 1$ joint degenerative change

\begin{tabular}{lccc} 
Characteristic & Mobile & Ankylosed & $p$-value \\
No. of patients & 62 & 24 & \\
Sex (male) & $53(85.5)$ & $17(70.8)$ & 0.118 \\
\hline Age at surgery (yr) & $70.5 \pm 11.7$ & $65.7 \pm 12$ & 0.094 \\
T1-T12 angle $\left(^{\circ}\right)$ & $57 \pm 18$ & $74 \pm 14$ & 0.004 \\
\hline T5-T12 angle $\left(^{\circ}\right)$ & $42 \pm 14$ & $56 \pm 11$ & 0.001 \\
X-angle $\left(^{\circ}\right)$ & $126 \pm 5$ & $136 \pm 5$ & $<0.001$ \\
\hline
\end{tabular}

Values are presented as number of patients (\%) or mean \pm standard deviation, unless otherwise stated. To identify differences between mobile and ankylosed group, a paired sample $t$-test and Fisher's exact test were used. 
in the supine position, because of the severe injuries in their spinal bone. Therefore, it was not possible to measure the fractured spine and non-fused mobile segments. We hope to be able to study the alignment of noninjured patients in future studies.

There were no significant differences observed between the age of patients in the mobile and ankylosed groups $(p=0.094)$. However, patients in the mobile group were slightly older than those in the ankylosed joint group. Due to the small sample size of this study, these results may vary.

Previous anatomical studies have described racial differences observed in the atlas and other craniocervical bones. It is therefore important to take such potential differences into consideration [17].

\section{Interpretation}

The definition of morphological change depended extensively on the investigator and selected measuring technique. Morphological changes were easily definable in patients with AS and a completely ankylosed C0-C1-C2 joint. However, in patients with AS and fusion of only one side of the joint, defining the morphological change was challenging (Fig. 5). The X-angle was a very useful tool in quantifying morphological change in such cases. The $\mathrm{X}$-angle was increased in cases with a collapsed and fused C0-C1-C2 joint. Koller et al. [18] reported the importance of the axial, sagittal, and coronal CT-reconstruction in revealing deformations and rotation of the OAA joint.

The fractures are often caused by low-energy impacts and are highly unstable due to the long lever arms produced by stiffness of the largely ankylosed spinal column. Therefore, most fractures require long-segment posterior fixation [2]. In this study, patients with AS and fracture of the cervical spinal were treated with long posterior stabilization from the upper cervical to the upper thoracic spine using a screw-rod system [19]. In patients with a fused C0-C1-C2 joint or large X-angle, extension of the instrumentation to the cranial bone was comparable in a strong construct.

Scheer et al. [9] reported that increased TK is correlated with increased cervical lordosis, and the Pearson coefficient $(r)$ value between the cervical and thoracic alignment was -0.51 . Lee et al. [10] reported sequential correlations between adjacent segments and demonstrated the correlation between the $\mathrm{C} 2-\mathrm{C} 7$ and $\mathrm{C} 0-\mathrm{C} 2$ angles

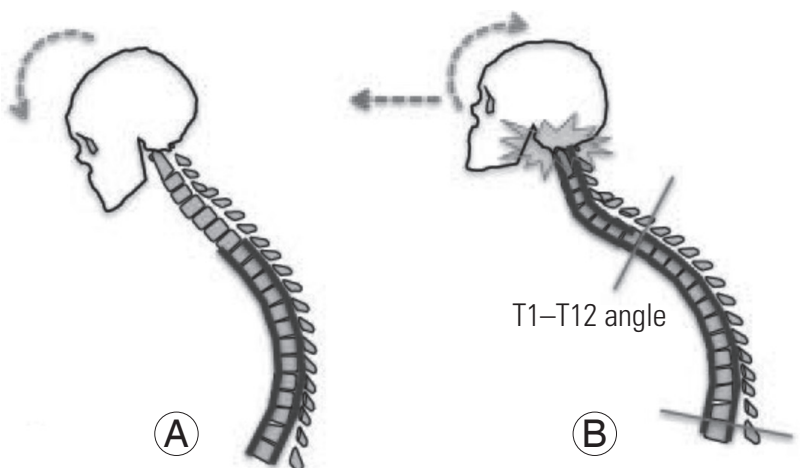

Fig. 6. (A, B) Schematic drawing illustrating the linkage of correlation from the thoracic kyphosis to the cranio-cervical parameters, $X$-angle, showed correlation. During progression of thoracic hyperkyphosis, an inclination of the occipitocervical joint occurred. The occipitocervical joints were forced into hyperlordosis to maintain the horizontal gaze. This may lead to secondary osteoarthritis and discomfort in the occipitoatlantoaxial joint.

Table 3. The intra- and inter-class reliabilities for $X$-angle measurement were very high suggesting a high accuracy of the measurement technique

\begin{tabular}{lccc} 
& Reliability & $\begin{array}{c}95 \% \text { confidence } \\
\text { interval }\end{array}$ & $p$-value \\
Intra-rater class & 0.945 & $0.907-0.967$ & $<0.001$ \\
Inter-rater class & 0.926 & $0.888-0.952$ & $<0.001$ \\
\hline
\end{tabular}

(the $r$ value was -0.547). In patients with AS and thoracic hyperkyphosis, the head was bending down. However, the occipitocervical joint was held in hyperlordosis to obtain a horizontal gaze. The compensatory hyperextension may lead to accelerated degeneration of the OAA joints. Furthermore, the OAA joint was collapsed and fused in patients with AS (Fig. 6). Finally, there were correlations detected between TK and the X-angle $(r=0.338, p=0.02)$.

The ICC and interclass reliabilities for the X-angle measurements were very high (0.95 and 0.93 , respectively) suggesting a strong correlation and high accuracy of this measurement technique (Table 3 ).

\section{Generalizability}

We used the new indicator X-angle in patients with AS and craniocervical arthritic or fused joints. Therefore, the $\mathrm{X}$-angle will be useful for the detection of occipitocervical diseases, such as rheumatoid arthritis [20], because this $\mathrm{X}$-angle increased in step with the vertical destruction of the C0-C1-C2 joints. Furthermore, patients with high Xangle values are often characterized by an abnormal cra- 
niocervical junction [21]. Therefore, it would be necessary to consider an availability of high riding vertebral artery in such patients.

\section{Conclusions}

A fused $\mathrm{C} 0-\mathrm{C} 1$ joint in patients with AS was related to thoracic hyperkyphosis. The $\mathrm{X}$-angle is a reliable tool for the quantification of morphological change in the $\mathrm{C} 0-\mathrm{C} 1$ C2 joint. These findings will improve our understanding of challenges in patients with AS related to cervical fractures, occipitocervical degenerative change, and strategy of surgical treatment.

\section{Conflict of Interest}

No potential conflict of interest relevant to this article was reported. NM and $\mathrm{AC}$ have no competing interests. $\mathrm{CO}$ and YR have gave paid lectures on spinal surgery topics unrelated to this work.

\section{Acknowledgments}

The authors thank the AOSpine and Uppsala University for hosting the first authors during their clinical fellowship. Furthermore the authors acknowledge H. Koller for their involvement in the development of this novel method. And this study was supported by the AOSpine Asia Pacific and AOSpine Europe travelling fellowships.

\section{References}

1. Olerud C, Frost A, Bring J. Spinal fractures in patients with ankylosing spondylitis. Eur Spine J 1996;5:51-5.

2. Westerveld LA, Verlaan JJ, Oner FC. Spinal fractures in patients with ankylosing spinal disorders: a systematic review of the literature on treatment, neurological status and complications. Eur Spine J 2009;18:145-56.

3. Heyde CE, Fakler JK, Hasenboehler E, et al. Pitfalls and complications in the treatment of cervical spine fractures in patients with ankylosing spondylitis. Patient Saf Surg 2008;2:15.

4. Schoenfeld AJ, Harris MB, McGuire KJ, Warholic N, Wood KB, Bono CM. Mortality in elderly patients with hyperostotic disease of the cervical spine after fracture: an age- and sex-matched study. Spine J 2011;11:257-64.

5. Robinson Y, Robinson AL, Olerud C. Complications and survival after long posterior instrumentation of cervical and cervicothoracic fractures related to ankylosing spondylitis or diffuse idiopathic skeletal hyperostosis. Spine (Phila Pa 1976) 2015;40:E227-33.

6. Goh L, Samanta A. A systematic MEDLINE analysis of therapeutic approaches in ankylosing spondylitis. Rheumatol Int 2009;29:1123-35.

7. Robinson Y, Sandén B, Olerud C. Increased occurrence of spinal fractures related to ankylosing spondylitis: a prospective 22-year cohort study in 17,764 patients from a national registry in Sweden. Patient Saf Surg 2013;7:2.

8. Simmons EH. Kyphotic deformity of the spine in ankylosing spondylitis. Clin Orthop Relat Res 1977;(128):65-77.

9. Scheer JK, Tang JA, Smith JS, et al. Cervical spine alignment, sagittal deformity, and clinical implications: a review. J Neurosurg Spine 2013;19:141-59.

10. Lee SH, Kim KT, Seo EM, Suk KS, Kwack YH, Son ES. The influence of thoracic inlet alignment on the craniocervical sagittal balance in asymptomatic adults. J Spinal Disord Tech 2012;25:E41-7.

11. Covaro AA, Manabe N, Bobinski L, Olerud C, Robinson Y. The importance of the occipitocervical area in patients with ankylosing spondylitis analysis of a cohort of 86 cervical fractures in surgically treated patients. J Craniovertebr Junction Spine 2017;8:3747.

12. Benchimol EI, Smeeth L, Guttmann A, et al. The Reporting of Studies Conducted Using Observational Routinely-Collected Health Data (RECORD) statement. PLoS Med 2015;12:e1001885.

13. Zanoli G, Nilsson LT, Stromqvist B. Reliability of the prospective data collection protocol of the Swedish Spine Register: test-retest analysis of 119 patients. Acta Orthop 2006;77:662-9.

14. Caron T, Bransford R, Nguyen Q, Agel J, Chapman J, Bellabarba C. Spine fractures in patients with ankylosing spinal disorders. Spine (Phila Pa 1976) 2010;35:E458-64.

15. Shrout PE, Fleiss JL. Intraclass correlations: uses in assessing rater reliability. Psychol Bull 1979;86:420-8.

16. Fleiss JL. Reliability of measurement. In: Fleiss JL, editor. The design and analysis of clinical experiments. 
Toronto: Wiley; 1986. p. 1-32.

17. Gosavi SN, Vatsalaswamy P. Morphometric study of the atlas vertebra using manual method. Malays Orthop J 2012;6:18-20.

18. Koller H, Acosta F, Forstner R, et al. C2-fractures: part II. a morphometrical analysis of computerized atlantoaxial motion, anatomical alignment and related clinical outcomes. Eur Spine J 2009;18:1135-53.

19. Carter S, Lories RJ. Osteoporosis: a paradox in ankylosing spondylitis. Curr Osteoporos Rep 2011;9:1125.
20. Tanouchi T, Shimizu T, Fueki K, et al. Neurological improvement and prognosis after occipito-thoracic fusion in patients with mutilating-type rheumatoid arthritis. Eur Spine J 2012;21:2506-11.

21. Wajanavisit W, Lertudomphonwanit T, Fuangfa P, Chanplakorn P, Kraiwattanapong C, Jaovisidha S. Prevalence of high-riding vertebral artery and morphometry of C2 pedicles using a novel computed tomography reconstruction technique. Asian Spine J 2016;10:1141-8. 